\title{
Anticoagulation Resumption After Colonic Polypectomy: Predicting Prime Post-procedural Timing
}

\author{
Natalia Causada Calo ${ }^{1} \cdot$ Jeffrey Mosko ${ }^{2}$ \\ Accepted: 23 November 2021 / Published online: 20 January 2022 \\ (c) The Author(s), under exclusive licence to Springer Science+Business Media, LLC, part of Springer Nature 2021
}

Colonoscopic detection and removal of adenomas reduces colorectal cancer incidence and associated mortality [1]. Though colonoscopy is considered a safe procedure, it is associated with a risk of bleeding when polypectomy is performed. Though the risk of post-polypectomy bleeding (PPB) is $1 \%$ in patients without risk factors [2], it is 1.8-8\% in patients receiving antiplatelet or anticoagulation therapy [3]. Other factors that can influence the risk of PPB include comorbidities such as renal and cardiovascular disease, polyp size and morphology, and polypectomy technique [4]. For instance, in patients requiring complex resections with techniques such as wide-field endoscopic mucosal resection, the risk of bleeding was reported to be $6.2 \%$, with repeat colonoscopy required in half of the patients [5]. Importantly, the highest bleeding risk is present within $48 \mathrm{~h}$ of the initial polypectomy.

Data obtained from large population-based studies indicate that the risk of thrombotic events is significant when anticoagulation is interrupted, with consequent significant morbidity and mortality [6]. Furthermore, data from observational cohort studies also suggest that anticoagulation resumption after an episode of gastrointestinal bleeding is associated with a lower risk of thrombosis and death, without a higher risk of recurrent bleeding [7]. The current knowledge regarding the risk of PPB in patients receiving anticoagulation, and how timing for anticoagulation resumption impacts outcomes in these patients is surprisingly limited. As the population ages, the use of anticoagulants has become more prevalent. Older age increases the burden of cardiovascular disease and other comorbidities, as well as the likelihood of polypectomy and post-polypectomy

Natalia Causada Calo

ncalo@toh.ca

1 Gastroenterology Division, The Ottawa Hospital, University of Ottawa, Ottawa, Canada

2 Gastroenterology Division, St. Michael's Hospital, University of Toronto, Toronto, Canada bleeding [8]. Furthermore, gastrointestinal bleeding in patients receiving antithrombotics has been associated with inferior outcomes [3]. To date, recommendations about the peri-endoscopic management of anticoagulation are largely based on expert opinion rather than on high-quality data. Current guidelines [2] do not integrate details regarding age, comorbidities, polypectomy technique (e.g., EMR vs conventional polypectomy), or polyp morphology to inform decision making. Understanding how risk factors for PPB interact is key to ponder the risks of bleeding and thrombosis and hence determine safe timing for anticoagulation resumption.

In this issue of Digestive Diseases and Sciences, Chebaa and colleagues report the results of a cohort study assessing the association between the timing of anticoagulation resumption and clinical outcomes in patients undergoing polypectomy [9]. The cohort was created as a post hoc analysis of a randomized clinical trial [10]. The authors included 437 patients who were chronically anticoagulated, interrupted the anticoagulation for the procedure, and resumed it post-polypectomy. The aims of the study were twofold: First they identified factors associated with a delay in 2 days or more in the resumption of anticoagulation. These factors were primarily related to the polyps themselves and polypectomy technique: The presence of complex, multiple, and right-sided polyps, the use of hot snare, fulguration, and piecemeal technique, and the use of thienopyridines were more prevalent in the late resumption group. Second, the authors assessed the association between early ( $<2$ days) versus late ( $>2$ days) anticoagulation resumption and risk of PPB and thromboembolic events. After adjusting for baseline confounding, the authors did not observe an association between timing of anticoagulation resumption and PPB. Regarding the risk of thrombosis, the authors report-in an unadjusted analysis - no thromboembolic events in the early resumption group and $2.3 \%$ of thromboembolic events in the late resumption group. Finally, while the timing of resumption of anticoagulation was not associated with the risk of 
bleeding, the authors observed that polyp size of $>1 \mathrm{~cm}$ and the use of fulguration were associated with a higher risk of PPB.

The study by Chebaa and colleagues builds upon previous research in the field and highlights important findings and limitations of the current body of literature [11]. First, as suggested by these findings, the main determinants of decisions surrounding the optimal timing of anticoagulation seem to be related to characteristics of the polypectomy itself. Whereas most of these variables appear to have face validity, it is unclear whether these factors were explicitly considered by clinicians while making post-procedure recommendations. Based on their findings, both polyp size and the use of fulguration should likely be considered in the decision-making process, given that these were associated with a higher risk of PPB. Interestingly, the use of thienopyridines was the only variable associated with a delay in anticoagulation that was not an aspect of the polypectomy. Patient demographics, comorbidities, and clinical scoring systems used to predict the risk of bleeding and thrombosis were not associated with a delayed resumption in anticoagulation. Overall, these findings highlight the underlying complexity of these decisions and the current profound limitations in understanding the factors that truly drive these judgements.

The second objective of the study aimed to assess the associations of early versus late resumption and clinical outcomes such as bleeding and thrombosis. The clinical dilemma of when to appropriately restart anticoagulation after a polypectomy and the attempts to balance the risks of bleeding and thrombosis have been extensively researched $[3,10]$. The observational nature of these data and the fact that the "strategies" of early versus late anticoagulation are not randomly allocated suggests that these two populations are likely very different at baseline. Even after adjusting for known confounders that might help mitigate the risk of treatment indication bias, there may still be unknown factors that render these two groups essentially non-comparable. Notwithstanding these limitations, the study conducted by Chebaa and colleagues remains an important contribution to the field. The risk of bleeding complications in patients that restarted the anticoagulation early was $<1 \%$ and with no observed thromboembolic events in this group. Despite the aforementioned challenges to draw conclusions about the efficacy and safety of these interventions, this study provides a reasonable estimate of the actual risk of complications in a population that resumed anticoagulation early, and perhaps helps reassure clinicians who elect to follow this strategy.

It is also important to mention that there are certain subgroups of patients to which the findings of this study do not apply. For example, these cannot be extrapolated to patients in whom anticoagulation is continued (i.e., patients with prosthetic heart valves) since the risk of thrombosis is considerably higher, or to patients with vary large and complex polyps, who were likely underrepresented in this study and for whom timing of anticoagulation resumption may be associated with a different risk of PPB.

In summary, the study by Chebaa et al. highlights important factors underlying the decision regarding as to when to restart anticoagulation in patients undergoing polypectomy. Furthermore, it provides reasonable estimates of the frequency of complications such as bleeding and thrombotic events. Finally, and perhaps most importantly, it highlights some of the limitations that the current body of literature has in answering the clinical question with regard to optimal timing of resumption of anticoagulation. This clinical question should be evaluated by carefully balancing the risk of bleeding and thrombosis in order to devise a strategy that translates into superior patient outcomes and should ideally be answered with a randomized clinical trial.

\section{References}

1. Zauber AG, Winawer SJ, O'Brien MJ et al. Colonoscopic polypectomy and long-term prevention of colorectal-cancer deaths. N Engl J Med 2012;366:687-696. https://doi.org/10.1056/NEJMo a1100370.

2. Acosta RD, Abraham NS, Chandrasekhara V et al. The management of antithrombotic agents for patients undergoing GI endoscopy. Gastrointest Endosc 2016;83:3-16. https://doi.org/10. 1016/j.gie.2015.09.035.

3. Abraham NS. Antiplatelets, anticoagulants, and colonoscopic polypectomy. Gastrointest Endosc 2020;91:257-265. https://doi.org/ 10.1016/j.gie.2019.09.033.Antiplatelets.

4. Sawhney MS, Salfiti N, Nelson DB, Lederle FA, Bond JH. Risk factors for severe delayed postpolypectomy bleeding. Endoscopy 2008;75:115-119.

5. Burgess NG, Metz AJ, Williams SJ et al. Risk factors for intraprocedural and clinically significant delayed bleeding after widefield endoscopic mucosal resection of large colonic lesions. Clin Gastroenterol Hepatol 2014;12:651-661.e3. https://doi.org/10. 1016/j.cgh.2013.09.049.

6. Raunso J, Selmer C, Olesen JB et al. Increased short-term risk of thrombo-embolism or death after interruption of warfarin treatment in patients with atrial fibrillation. Eur Heart $J$ 2012;33:1886-1892. https://doi.org/10.1093/eurheartj/ehr454.

7. Witt DM, Delate T, Garcia D et al. Risk of thromboembolism, recurrent hemorrhage, and death after warfarin therapy interruption for gastrointestinal tract bleeding. Arch Intern Med 2012;172:1484-1491. https://doi.org/10.1001/archinternmed. 2012.4261.

8. Causada-Calo N, Bishay K, Albashir S, Al Mazroui A, Armstrong D. Association between age and complications after outpatient colonoscopy. JAMA Netw Open 2020;3:1-13. https://doi.org/10. 1001/jamanetworkopen.2020.8958.

9. Chebaa et al Timing of resumption of anticoagulation after polypectomy and frequency of post-procedural complications: a posthoc analysis. Dig Dis Sci. (Epub ahead of print). https://doi.org/ 10.1007/s10620-021-07341-3.

10. Feagins LA, Smith AD, Kim D et al. Efficacy of prophylactic hemoclips in prevention of delayed post-polypectomy 
bleeding in patients with large colonic polyps. Gastroenterology 2019;157:967-976.e1. https://doi.org/10.1053/j.gastro.2019.05. 003.

11. Shalman D, Gerson LB. Systematic review with meta-analysis: the risk of gastrointestinal haemorrhage post-polypectomy in patients receiving anti-platelet, anti-coagulant and/or thienopyridine medications. Aliment Pharmacol Ther 2015;42:949-956. https://doi. org/10.1111/apt.13367.
Publisher's Note Springer Nature remains neutral with regard to jurisdictional claims in published maps and institutional affiliations. 